Journal of Applied AnALysis

Vol. 8, No. 1 (2002), pp. 141-151

\title{
ON THE STATIONARY FLOW OF THE POWER LAW FLUID IN 2D
}

\author{
W. SADOWSKI
}

Received August 8, 2000 and, in revised form, June 4, 2001

\begin{abstract}
We consider the stationary flow of a heat conducting Power Law shear thinning fluid in a bounded domain in $\mathbb{R}^{2}$. We present an elementary proof of existence of at least one weak solution.
\end{abstract}

\section{Introduction}

Various mathematical aspects of the flow of a heat conducting incompressible nonNewtonian fluid was studied recently by many author ([2], [3], [4], [5]). A considerable attention was paid to the flow of a very important class of nonNewtonian fluids called Power Law fluids.

The general governing system of equations for the flow of heat conducting incompressible fluids consists of the following equations:

$$
\begin{gathered}
\operatorname{div} u=0, \\
\rho \frac{\partial u_{i}}{\partial t}=-\rho u_{j} \frac{\partial u_{i}}{\partial x_{j}}+f_{i} \rho+\operatorname{div} T_{i} \quad i=1,2, \ldots, d, \\
\rho \frac{\partial \theta}{\partial t}=-\rho u_{j} \frac{\partial \theta}{\partial x_{j}}+\frac{\partial u_{i}}{\partial x_{j}} T_{i j}-\operatorname{div}(-K \nabla \theta) .
\end{gathered}
$$

2000 Mathematics Subject Classification. 35Q35, 76D03.

Key words and phrases. non-Newtonian fluid, existence, fixed point argument.

ISSN 1425-6908 C Heldermann Verlag. 
Here $u=\left(u_{1}, u_{2}, \ldots, u_{d}\right)$ is the velocity of the fluid, $\theta$ is the temperature, $T_{i}$ is the $i$-th column of the stress tensor $T, f=\left(f_{1}, f_{2}, \ldots, f_{d}\right)$ represents external forces, $K$ is the thermal conductivity and $d$ is the space dimension. In special case of the Power Law fluids the stress tensor is of the form

$$
T_{i j}=k(\theta)|e(u)|^{r-2} e_{i j}(u)-\delta_{i j} p,
$$

where $k(\theta)$ is a function of temperature, $\delta$ is the Kronecker's symbol and $e(u)$ is a matrix defined by

$$
e_{i j}=\frac{1}{2}\left(\frac{\partial u_{i}}{\partial x_{j}}+\frac{\partial u_{j}}{\partial x_{i}}\right)
$$

The parameter $r$ is a real number bigger than 1 . If $1<r<2$ then the fluid is shear thinning. If $r=2$ the fluid is Newtonian and if $r>2$ we have the case of the shear thickening fluid.

In this paper we consider the stationary flow of incompressible Power Law shear thinning fluid in a 2 -dimensional bounded domain $\Omega$. For simplicity we set $\rho=K=1$. Then the system (1.1)-(1.3) takes the form:

$$
\begin{array}{ll}
\operatorname{div} u=0 & \text { in } \Omega, \\
-\frac{\partial}{\partial x_{j}}\left(k(\theta)|e(u)|^{r-2} e_{i j}(u)\right)-\frac{\partial p}{\partial x_{i}}+u_{j} \frac{\partial u_{i}}{\partial x_{j}}=f_{i} & (i=1,2) \\
-\triangle \theta+u_{j} \frac{\partial \theta}{\partial x_{j}}=\frac{\partial u_{i}}{\partial x_{j}}\left(k(\theta)|e(u)|^{r-2} e_{i j}(u)-\delta_{i j} p\right) & \text { in } \Omega, \\
\left.u\right|_{\partial \Omega}=\left.u_{0} \quad \theta\right|_{\partial \Omega}=\theta_{0} . &
\end{array}
$$

The existence of solutions for this system with nonhomogeneous Dirichlet boundary conditions was proved in [4]. In a special case of this system for $1<r<2$ and with homogeneous boundary conditions we prove the existence of solutions in a more elementary way. We also get slightly better regularity of temperature since our solution belongs not only to each of the Sobolev spaces $W^{1, s}(\Omega), 1 \leq s<2$, but also to Sobolev space $H^{1}(\Omega)$.

The uniqueness of solutions for the system (1.5)-(1.8) remains an open problem. Only for the system without a convection term in dynamical equation the uniqueness of solutions was proved in [2].

\section{Notation}

In this paper we use the following notation:

$\Omega$ - an open bounded set of a class $C^{2}, \Omega \subset \mathbb{R}^{2}$

$L^{q}$ - the usual Lebesgue's space $L^{q}(\Omega)$, with the standard norm denoted by $|\cdot|_{q}$ 
$W_{0}^{1, r}$ - closure of the set of smooth functions with compact support in $\Omega$ (denoted by $C_{0}^{\infty}(\Omega)$ ) in the norm

$$
\|u\|_{r}=\left(\int_{\Omega}|u|^{r}+|\nabla u|^{r} d x\right)^{1 / r}
$$

$\tilde{V}=\left\{u \in C_{0}^{\infty}(\Omega)^{2}: u=\left(u_{1}, u_{2}\right), \operatorname{div} u=0\right.$ in $\left.\Omega\right\}$ $V^{1, r}=$ closure of $\tilde{V}$ in $W_{0}^{1, r}$.

\section{Setting of the problem}

In this section we define a weak form of the system (1.5)-(1.7) with the homogeneous boundary conditions. Multiplying the $i$-th equation of (1.6) by smooth function $\phi_{i} \in C_{0}^{\infty}, i=1,2$, div $\left(\phi_{1}, \phi_{2}\right)=0$ in $\Omega$ we obtain after adding equations and integration by parts:

$$
\begin{gathered}
\int_{\Omega} k(\theta)|e(u)|^{r-2} e_{i j}(u) e_{i j}(\phi)-\int_{\Omega} \frac{\partial p}{\partial x_{i}} \phi_{i}+\int_{\Omega} u_{j} \frac{\partial u_{i}}{\partial x_{j}} \phi_{i}=\int_{\Omega} f_{i} \cdot \phi_{i} \\
i=1,2 .
\end{gathered}
$$

Similarly, for (1.7) and $\xi \in C_{0}^{\infty}(\Omega)$ we obtain:

$$
\begin{aligned}
\int_{\Omega} \nabla \theta \cdot \nabla \xi+\int_{\Omega} u_{j} \frac{\partial \theta}{\partial x_{j}} \xi= & \int_{\Omega} \frac{\partial u_{i}}{\partial x_{j}} k(\theta)|e(u)|^{r-2} e_{i j}(u) \xi \\
& -\int_{\Omega} \frac{\partial u_{i}}{\partial x_{j}} \delta_{i j} p \xi .
\end{aligned}
$$

Since the first term on the right is equal to $\int_{\Omega} k(\theta)|e(u)|^{r} \xi$ and the second term vanishes we have:

$$
\int_{\Omega} \nabla \theta \cdot \nabla \xi+\int_{\Omega} u_{j} \frac{\partial \theta}{\partial x_{j}} \xi=\int_{\Omega} k(\theta)|e(u)|^{r} \xi .
$$

The term $k(\theta)|e(u)|^{r}$ naturally belongs to $L^{1}$, when $k$ is a bounded function and $u$ belongs to Sobolev space $W^{1, r}$. However, using again the equation of dynamics, we get more convenient expression for this term. Multiplying the $i$-th equation of (1.6) by $u_{i} \cdot \xi$, adding equations and integrating over $\Omega$ we get:

$$
\begin{aligned}
\int_{\Omega} k(\theta)|e(u)|^{r} \xi= & \int_{\Omega}(f \cdot u) \xi-\int_{\Omega} u_{j} \frac{\partial u_{i}}{\partial x_{j}} u_{i} \xi-\int_{\Omega} p u \cdot \nabla \xi \\
& -\int_{\Omega} k(\theta)|e(u)|^{r-2} e_{i j}(u) \cdot \frac{\partial \xi}{\partial x_{j}} u_{i} .
\end{aligned}
$$


Finally, the weak form of (1.7), which we shall use is (cf. [5]):

$$
\begin{aligned}
\int_{\Omega} \nabla \theta \cdot \nabla \xi+\int_{\Omega} u_{j} \frac{\partial \theta}{\partial x_{j}} \xi= & \int_{\Omega}(f \cdot u) \xi-\int_{\Omega} u_{j} \frac{\partial u_{i}}{\partial x_{j}} u_{i} \xi-\int_{\Omega} p u \cdot \nabla \xi \\
& -\int_{\Omega} k(\theta)|e(u)|^{r-2} e_{i j}(u) \cdot \frac{\partial \xi}{\partial x_{j}} u_{i} .
\end{aligned}
$$

Definition 3.1. We call a pair of functions $(u, \theta) \in V^{1, r} \times H_{0}^{1}$ a weak solution of (1.5)-(1.8) with $u_{0}=\theta_{0}=0$ if (3.1) holds for all $\phi \in V^{1, r}$ and (3.5) holds for all $\xi \in H_{0}^{1}$.

The aim of this paper is to prove

Theorem 3.1. Let $\Omega \subset \mathbb{R}^{2}$ be a bounded set of class $C^{2}$. If $r \in(3 / 2,2)$, the function $k(\theta)$ is positive, bounded and separated from 0 :

$$
k(x) \geq k_{1}>0 \quad \forall x \in \mathbb{R}
$$

and

$$
f \in L^{2 r /(3 r-2)+\varepsilon}
$$

for some $\varepsilon>0$, then there exists a weak solution of the system (1.5)-(1.7) with homogenous boundary condition in the sense of Definition 3.1.

\section{Auxiliary results}

Below we state some lemmas which we will need in the proof of the main theorem.

Lemma 4.1 ([8]). For $u, w, s \in V$ and

$$
b(u, w, s)=\int_{\Omega} u_{j} \frac{\partial w_{i}}{\partial x_{j}} s_{i}
$$

we have: $b(u, w, s)=-b(u, s, w)$.

Lemma $4.2([7])$. For $x, y \in \mathbb{R}^{n}$ the following inequality holds:

$$
\left(|x|^{r-2} x-|y|^{r-2} y\right) \cdot(x-y) \geq \frac{|x-y|^{2}}{(|x|+|y|)^{2-r}} \quad \text { for } \quad 1<r<2 .
$$


Lemma 4.3. The expression

$$
\|u\|_{*}=\left(\int_{\Omega}|e(u)|^{r}\right)^{1 / r}
$$

is a norm in $V^{1, r}$ equivalent to the standard norm in $V^{1, r}$, introduced above.

Lemma 4.4. For $r>3 / 2$ we have compact imbedding $W^{1, r}$ in $L^{2 r /(r-1)+\varepsilon}$ for some $\varepsilon>0$.

\section{The proof of existence}

We start with the definition of an operator $K: V^{1, r} \times H_{0}^{1} \rightarrow V^{1, r} \times H_{0}^{1}$.

Definition 5.1. For the operator $K: V^{1, r} \times H_{0}^{1} \rightarrow V^{1, r} \times H_{0}^{1}$ we have

$$
K(u, \theta)=\left(u^{*}, \theta^{*}\right)
$$

if and only if the following equalities hold:

$$
\begin{aligned}
\int_{\Omega} k(\theta)\left|e\left(u^{*}\right)\right|^{r-2} e_{i j}\left(u^{*}\right) e_{i j}\left(\phi_{i}\right)+\int_{\Omega} u_{j} \frac{\partial u_{i}^{*}}{\partial x_{j}} \phi_{i} & =\int_{\Omega} f_{i} \cdot \phi_{i} \\
i & =1,2
\end{aligned}
$$

for all $\phi$ in $V^{1, r}$ and

$$
\begin{aligned}
\int_{\Omega} \nabla \theta^{*} \cdot \nabla \xi+\int_{\Omega} u_{j}^{*} \frac{\partial \theta^{*}}{\partial x_{j}} \xi & =\int_{\Omega} f \cdot u^{*} \xi-\int_{\Omega} u_{j}^{*} \frac{\partial u_{i}^{*}}{\partial x_{j}} u_{i}^{*} \xi-\int_{\Omega} p u^{*} \cdot \nabla \xi \\
& -\int_{\Omega} k(\theta)\left|e\left(u^{*}\right)\right|^{r-2} e_{i j}\left(u^{*}\right) \cdot \frac{\partial \xi}{\partial x_{j}} u_{i}^{*}
\end{aligned}
$$

for all $\xi \in H_{0}^{1}$, where $p$ is the pressure associated with equation (5.1).

Remark 5.1. In the equation (5.2) we need to know the corresponding pressure function $p$. This function is a unique (up to a constant) solution of (1.6) on $\Omega$. Moreover, $p \in L^{r /(r-1)}$ and the norm $|p|_{r /(r-1)}$ is bounded by the norm of

$$
-\frac{\partial}{\partial x_{j}}\left(k(\theta)|e(u)|^{r-2} e_{i j}(u)\right)+u_{j} \frac{\partial u_{i}}{\partial x_{j}}-f_{i}
$$

in the dual space $\left(V^{1, r}\right)^{*}$. (For more details see [8] and [4].)

Lemma 5.1. The operator $K$ defined above is well defined. 
Proof. The existence of a unique solution $u^{*}$ to the equation (5.1) follows from Browder-Minty theorem. Indeed, the operator $A$ defined by

$$
\left(A\left(u^{*}\right), \phi\right)=\int_{\Omega} k(\theta)\left|e\left(u^{*}\right)\right|^{r-2} e_{i j}\left(u^{*}\right) e_{i j}(\phi)+\int_{\Omega} u_{j} \frac{\partial u_{i}^{*}}{\partial x_{j}} \phi
$$

where $u$ is given, is bounded, strictly monotonous, coercive and hemicontinuous. (For more details see [6].)

Now, we need to prove existence of a unique solution to the equation (5.2). We will use Lax-Milgram lemma. It is easy to see that for given $u^{*}$ the left hand side of (5.2) defines the form

$$
C(\theta, \xi)=\int_{\Omega} \nabla \theta \cdot \nabla \xi+\int_{\Omega} u^{*} \cdot \nabla \theta \xi
$$

which is coercive, bilinear and continuous on $H_{0}^{1} \times H_{0}^{1}$. All we need to prove is that the linear operator

$F(\xi)=\int_{\Omega}\left(f \cdot u^{*}\right) \xi-\int_{\Omega} u_{j}^{*} \frac{\partial u_{i}^{*}}{\partial x_{j}} u_{i}^{*} \xi-\int_{\Omega} p u^{*} \cdot \nabla \xi-\int_{\Omega} k(\theta)\left|e\left(u^{*}\right)\right|^{r-2} e_{i j}\left(u^{*}\right) \cdot \frac{\partial \xi}{\partial x_{j}} u_{i}^{*}$

is continuous on $H_{0}^{1}$. Using Lemmas 4.3, 4.4, Hölder's inequality and the Sobolev imbedding theorem (cf. [1]) we obtain the following estimates (here we use the assumption on $\Omega$ being two dimensional):

$$
\int_{\Omega}\left(f \cdot u^{*}\right) \xi \leq|f|_{\alpha} \cdot\left|u^{*}\right|_{2 r /(2-r)} \cdot|\xi|_{q} \leq C|| u^{*}||_{r} \cdot|f|_{\alpha} \cdot\|\xi\|_{2},
$$

where $\alpha=2 r /(3 r-2)+\delta, \delta>0$ and $q$ satisfies $1 / q+1 / \alpha+(2-r) / 2 r=1$,

$$
\int_{\Omega} u_{j}^{*} \frac{\partial u_{i}^{*}}{\partial x_{j}} u_{i}^{*} \xi \leq\left|u^{*}\right|_{\beta}^{2} \cdot\left|\nabla u^{*}\right|_{r}|\xi|_{\gamma} \leq C|| u^{*}\left\|_{r}^{3} \cdot\right\| \xi \|_{2},
$$

where $\beta=2 r /(r-1)+\varepsilon, \varepsilon>0$ and $\gamma$ satisfies $1 / \beta+1 / \gamma+1 / r=1$,

$$
\begin{gathered}
\int_{\Omega} p \cdot u^{*} \cdot \nabla \xi \leq|p|_{r /(r-1)} \cdot\left|u^{*}\right|_{2 r /(r-1)} \cdot|\nabla \xi|_{2} \leq\left. C|p|_{r /(r-1)} \cdot\left\|u^{*}\right\|\right|_{r} \cdot\|\xi\|_{2} \\
\int_{\Omega} k(\theta)\left|e\left(u^{*}\right)\right|^{r-2} e_{i j}\left(u^{*}\right) \cdot \frac{\partial \xi}{\partial x_{j}} u_{i}^{*} \leq C_{0}\left|e\left(u^{*}\right)\right|_{r}^{r-1} \cdot\left|u^{*}\right|_{2 r /(r-1)} \cdot|\nabla \xi|_{2} \\
\leq C\left\|u^{*}\right\|_{r}^{r}\|\xi\|_{2} .
\end{gathered}
$$

Then we have:

$$
|F(\xi)| \leq C\left(\|\left. u^{*}\right|_{r},|p|_{r /(r-1)}, \Omega,|f|_{\alpha}\right) \cdot\|\xi\|_{2} .
$$

This finishes the proof. 
Lemma 5.2. There exists a closed ball $B=B(0, R)$ centered at zero and of radius $R$ in $V^{1, r} \times H_{0}^{1}$ such that operator $K$ maps $B$ into itself.

Proof. Setting $\phi=u^{*}$ in (5.1) we obtain:

$$
\int_{\Omega} k(\theta)\left|e\left(u^{*}\right)\right|^{r}+\int_{\Omega} u_{j} \frac{\partial u_{i}^{*}}{\partial x_{j}} u_{i}^{*}=\int_{\Omega} f_{i} \cdot u_{i}^{*} .
$$

According to lemma 4.1 the second term in equation above vanishes. Moreover, using lemma 4.3 and assumption that $k(\cdot)$ is separated from zero we obtain:

$$
k_{1} C\left\|u^{*}\right\|_{r}^{r} \leq|f|_{*}\left\|u^{*}\right\|_{r}
$$

where $|f|_{*}$ is the norm of function $f$ in the dual space $\left(V^{1, r}\right)^{*}$.

Then we have:

$$
\left\|u^{*}\right\|_{r} \leq C_{1}
$$

where $C_{1}$ is a constant depending only on $\Omega$, the norm $|f|_{*}$ and the constant $k_{1}$ which separates function $k$ from zero. Now setting $\xi=\theta^{*}$ in (5.2) we easily obtain:

$$
\left\|\theta^{*}\right\|_{2}^{2} \leq\left|F\left(\theta^{*}\right)\right|
$$

and according to (5.3) we have:

$$
\left\|\theta^{*}\right\|_{2} \leq C_{2}
$$

where $C_{2}$ depends on the norms of $\left\|u^{*}\right\|_{r}, p$ and $f$ and on $\Omega$. From this, the bound for $\left\|u^{*}\right\|_{r}$ and the Remark 5.1 we obtain that the solutions $\theta^{*}$ of (5.2) are also bounded by some constant $C\left(\Omega,|f|_{*}, k_{1}\right)$.

Lemma 5.3. The operator $K$ is weakly continuous in $V^{1, r} \times H_{0}^{1}$.

Proof. Let $u_{m} \rightarrow u$ weakly in $V^{1, r}$ and $\theta_{m} \rightarrow \theta$ weakly in $H_{0}^{1}$. We need to show that $u_{m}^{*} \rightarrow u^{*}$ weakly in $V^{1, r}$ and $\theta_{m}^{*} \rightarrow \theta^{*}$ weakly in $H_{0}^{1}$, where $K\left(u_{m}, \theta_{m}\right)=\left(u_{m}^{*}, \theta_{m}^{*}\right)$ and $K(u, \theta)=\left(u^{*}, \theta^{*}\right)$. Observe that since $u_{m}^{*}$ is bounded in $V^{1, r}$ it contains a subsequence weakly convergent to some $u_{+}^{*} \in$ $V^{1, r}$. We will show that $u_{+}^{*}=u^{*}$ following the idea presented in [5]. First, we set $\phi=u_{m}^{*}-u_{+}^{*}$ in (5.1) with $u^{*}$ replaced by $u_{m}^{*}$ and then we subtract $\int_{\Omega} k\left(\theta_{m}\right)\left|e\left(u *_{+}\right)\right|^{r-2} e_{i j}\left(u_{+}^{*}\right) e_{i j}\left(u_{m}^{*}-u_{+}^{*}\right)$ from both sides to obtain

$$
\int_{\Omega} k\left(\theta_{m}\right)\left(\left|e\left(u_{m}^{*}\right)\right|^{r-2} e_{i j}\left(u_{m}^{*}\right)-\left|e\left(u_{+}^{*}\right)\right|^{r-2} e_{i j}\left(u_{+}^{*}\right)\right) e_{i j}\left(u_{m}^{*}-u_{+}^{*}\right)
$$




$$
\begin{aligned}
& =-\int_{\Omega}\left(u_{m}\right)_{j} \frac{\partial\left(u_{m}^{*}\right)_{i}}{\partial x_{j}}\left(\left(u_{m}^{*}\right)_{i}-\left(u_{+}^{*}\right)_{i}\right)+\int_{\Omega} f \cdot\left(u_{m}^{*}-u_{+}^{*}\right) \\
& -\int_{\Omega} k\left(\theta_{m}\right)\left|e\left(u_{+}^{*}\right)\right|^{r-2} e_{i j}\left(u_{+}^{*}\right) e_{i j}\left(u_{m}^{*}-u_{+}^{*}\right) .
\end{aligned}
$$

Due to Lemma 4.2 and (3.6) the left hand side of (5.4) is not less than $k_{1} C_{1}\left\|u_{m}^{*}-u_{+}^{*}\right\|_{r}$ (for more details see [3]). We will show that the right hand side of (5.4) tends to zero as $m \rightarrow \infty$. We have, eventually for a subsequence,

$$
\begin{aligned}
& \int_{\Omega}\left(u_{m}\right)_{j} \frac{\partial\left(u_{m}^{*}\right)_{i}}{\partial x_{j}}\left(\left(u_{m}^{*}\right)_{i}-\left(u_{+}^{*}\right)_{i}\right) \\
& \leq\left.\left|u_{m}\right|_{2 r /(r-1)} \cdot|| u_{m}^{*}\right|_{r} \cdot\left|u_{m}^{*}-u_{+}^{*}\right|_{2 r /(r-1)} \rightarrow 0
\end{aligned}
$$

since $\left\|u_{m}^{*}\right\|_{r}$ is bounded (Lemma 5.2) and $\left\|u_{m}\right\|_{r}$ is bounded too (because of weak convergence of $u_{m}$ ) and, taking into account that $\Omega$ is a set on the plane and $3 / 2<r<2$, it follows from the Rellich-Kondrachov theorem that we can choose a subsequence strongly convergent in $L^{2 r /(r-1)}$. Moreover, from the weak convergence of $u_{m}^{*} \rightarrow u_{+}^{*}$ we get

$$
\int_{\Omega} f \cdot\left(u_{m}^{*}-u_{+}^{*}\right) \rightarrow 0 .
$$

To show that also the third term on the right hand side of (5.4) tends to zero, we split it into three terms below and we show that:

$$
\begin{gathered}
\int_{\Omega}\left[k\left(\theta_{m}\right)-k(\theta)\right]\left|e\left(u_{+}^{*}\right)\right|^{r-2} e_{i j}\left(u_{+}^{*}\right) e_{i j}\left(u_{m}^{*}\right) \rightarrow 0, \\
\int_{\Omega} k(\theta)\left|e\left(u_{+}^{*}\right)\right|^{r-2} e_{i j}\left(u_{+}^{*}\right) e_{i j}\left(u_{m}^{*}-u_{+}^{*}\right) \rightarrow 0, \\
\int_{\Omega}\left[k(\theta)-k\left(\theta_{m}\right)\right]\left|e\left(u_{+}^{*}\right)\right|^{r} \rightarrow 0 .
\end{gathered}
$$

To obtain the convergence (5.7) we observe that $\theta_{m} \rightarrow \theta$ almost everywhere for a subsequence. Indeed, the functions $\theta_{m}$ are bounded in $H_{0}^{1}$ and it follows from the Rellich-Kondrachov theorem that there exist a subsequence strongly convergent in $L^{2}$ and, eventually for another subsequence, we have convergence a.e. Since $k$ is continuous we have $k\left(\theta_{m}\right) \rightarrow k(\theta)$ a.e. Now, using Lebesgue's dominated convergence theorem, we get that $k\left(\theta_{m}\right)\left|e\left(u^{*}\right)\right|^{r-1}$ is, eventually for a subsequence, strongly convergent in $L^{r /(r-1)}$ and (5.7) follows from Hölder's inequality. The convergence in (5.8) is due to the weak convergence of $u_{m}^{*}$. Finally, (5.9) follows from a.e. convergence of $k\left(\theta_{m}\right)$ and 
Lebesgue's dominated convergence theorem. We showed that the right hand side of (5.4) tends to zero as $m$ tends to infinity. Since the left hand side is not less than $k_{1} C_{1}\left\|u_{m}^{*}-u_{+}^{*}\right\|_{r}$ we obtain that for a subsequence:

$$
u_{m}^{*} \rightarrow u_{+}^{*} \quad \text { strongly in } V^{1, r} .
$$

The strong convergence (5.10) and the weak convergence of $u_{m}$ allow us to pass to the limit in (5.1) with $u^{*}$ replaced by $u_{m}^{*}$ and conclude that $u_{+}^{*}$ satisfies the equation

$$
\begin{aligned}
\int_{\Omega} k(\theta)\left|e\left(u_{+}^{*}\right)\right|^{r-2} e_{i j}\left(u_{+}^{*}\right) e_{i j}\left(\phi_{i}\right)+\int_{\Omega} u_{j} \frac{\partial\left(u_{+}^{*}\right)_{i}}{\partial x_{j}} \phi_{i} & =\int_{\Omega} f_{i} \cdot \phi_{i} \\
i=1,2 . &
\end{aligned}
$$

Since this solution is unique, we get $u^{*}=u_{+}^{*}$.

Now we will show that $\theta_{m}^{*} \rightarrow \theta^{*}$ in $H_{0}^{1}$. Since $\theta_{m}^{*}$ is bounded, there exists a subsequence for which $\theta_{m}^{*} \rightarrow \theta_{+}^{*}$, weakly for some $\theta_{+}^{*} \in H_{0}^{1}$. We need to show that $\theta_{+}^{*}=\theta^{*}$, where $\theta^{*}$ is a solution to (5.2). For fixed $\xi \in H_{0}^{1}$ we have, due to the strong convergence of $u_{m}^{*}$ in $V^{1, r}$

$$
\begin{gathered}
\int_{\Omega}\left(f \cdot u_{m}^{*}\right) \xi \rightarrow \int_{\Omega}\left(f \cdot u^{*}\right) \xi \\
\int_{\Omega}\left(u_{m}^{*}\right)_{j} \frac{\partial\left(u_{m}\right)_{i}^{*}}{\partial x_{j}}\left(u_{m}^{*}\right)_{i} \xi \rightarrow \int_{\Omega}\left(u^{*}\right)_{j} \frac{\partial u_{i}^{*}}{\partial x_{j}}\left(u^{*}\right)_{i} \xi .
\end{gathered}
$$

Moreover, from the strong convergence of $u_{m}^{*}$ in $V^{1, r}$ and the weak convergence of $p_{m}$ in $L^{r /(r-1)}(\Omega)$ (see Remark 5.1) it follows that

$$
\int_{\Omega} p_{m} u_{m}^{*} \cdot \nabla \xi \rightarrow \int p u^{*} \nabla \xi .
$$

Finally, due to Lebesgue's dominated convergence theorem and almost everywhere convergence for a subsequence of $u_{m}^{*}$ and $k\left(\theta_{m}\right)$ we have

$$
\begin{aligned}
& \int_{\Omega} k\left(\theta_{m}\right)\left|e\left(u_{m}^{*}\right)\right|^{r-2} e_{i j}\left(u_{m}^{*}\right) \cdot \frac{\partial \xi}{\partial x_{j}}\left(u_{m}^{*}\right)_{i} \\
& \rightarrow \int_{\Omega} k(\theta)\left|e\left(u^{*}\right)\right|^{r-2} e_{i j}\left(u^{*}\right) \cdot \frac{\partial \xi}{\partial x_{j}} u_{i}^{*}
\end{aligned}
$$


From (5.12)-(5.15), the strong convergence of $u_{m}^{*}$ in $V^{1, r}$ and the weak convergence of $\theta_{m}^{*}$ in $H_{0}^{1}$ follows that we can pass to the limit in the equation

$$
\begin{aligned}
& \int_{\Omega} \nabla \theta_{m}^{*} \cdot \nabla \xi+\int_{\Omega}\left(u_{m}\right)_{j}^{*} \frac{\partial \theta_{m}^{*}}{\partial x_{j}} \xi=\int_{\Omega} f \cdot u_{m}^{*} \xi-\int_{\Omega}\left(u_{m}\right)_{j}^{*} \frac{\partial\left(u_{m}\right)_{i}^{*}}{\partial x_{j}}\left(u_{m}\right)_{i}^{*} \xi \\
& -\int_{\Omega} p_{m} u_{m}^{*} \cdot \nabla \xi-\int_{\Omega} k\left(\theta_{m}\right)\left|e\left(u_{m}^{*}\right)\right|^{r-2} e_{i j}\left(u_{m}^{*}\right) \cdot \frac{\partial \xi}{\partial x_{j}}\left(u_{m}\right)_{i}^{*}
\end{aligned}
$$

and conclude that $\theta_{+}^{*}$ is a solution of the equation (5.2). Since this solution is unique we get $\theta_{+}^{*}=\theta^{*}$.

Summarizing the results of last three lemmas we obtain - due to SchauderTichonov theorem — the Theorem 3.1.

Acknoledgments. The author is grateful to Professor Grzegorz Lukaszewicz for helpfull discussions and to the Referees for important remarks and suggestions that helped to improve the paper.

\section{References}

[1] Adams, R. A., Sobolev Spaces, Academic Press, New York, 1975.

[2] Baranger, J., Mikelić, A., Stationary solutions to a quasi-Newtonian flow with viscous heating, Math. Models Methods Appl. Sci. 5(6) (1995), 725-738.

[3] Boukrouche, M., Łukaszewicz, G., The stationary Stefan problem with convection governed by nonlinear Darcy's law, Math. Methods Appl. Sci. 22 (1999), 563-585.

[4] Clopeau, Th., Mikelić, A., Nonstationary flows with viscous heating effects, Elasticité, viscoélasticité et contrōle optimal (Lyon, 1995), 55-63, ESAIM Proc. 2, Soc. Math. Appl. Indust., Paris, 1997.

[5] Gilbert, R. P., Shi, P., Nonisothermal, nonNewtonian Hele-Shaw flows. Part II, Nonlinear Anal. 27(5) (1996), 539-559.

[6] Lions, J. L., Quelques Methodes de Resolution des Problemes aux Limites Nonlineares, Dunod, Gauthier-Villars, Paris, 1969.

[7] Morrey, Ch. B., Multiple Integrals in the Calculus of Variations, Springer, Berlin, 1966.

[8] Temam, R., Navier-Stokes Equations. Theory and Numerical Analysis, Stud. Math. Appl. 2, North-Holland, Amsterdam, 1979.

[9] Wardi, S., A convergence result for an iterative method, for the equations of stationary quasi-Newtonian flow with temperature dependent viscosity, RAIRO Modél. Math. Anal. Numér. 32(4) (1998), 391-404. 
WiTOLD SADOWSKI

Institute of Applied Mathematics

WARSAW UNIVERSITY

BANACHA 22

02-097 WARSAW, POLAND

WITEKS@MIMUW.EDU.PL 